\title{
Análise florística das pteridófitas do Parque Nacional da Restinga de Jurubatiba, Rio de Janeiro, Brasil ${ }^{1}$
}

\author{
Marcelo Guerra Santos ${ }^{2,4}$, Lana da Silva Sylvestre ${ }^{3}$ e Dorothy Sue Dunn de Araujo ${ }^{4}$
}

Recebido em 30/06/2002. Aceito em 09/09/2003

\begin{abstract}
RESUMO - (Análise florística das pteridófitas do Parque Nacional da Restinga de Jurubatiba, Rio de Janeiro, Brasil). O Parque Nacional da Restinga de Jurubatiba, localizado no norte do Estado do Rio de Janeiro, abrange os municípios de Macaé, Carapebus e Quissamã. Foram registradas para a área de estudo 12 famílias com 21 gêneros e 32 espécies. As famílias encontradas foram Blechnaceae, Cyatheaceae, Davalliaceae, Dennstaedtiaceae, Dryopteridaceae, Gleicheniaceae, Lycopodiaceae, Polypodiaceae, Pteridaceae, Salviniaceae, Schizaeaceae e Thelypteridaceae. Dessas, as mais representativas em número de espécies foram Polypodiaceae (5 spp.) e Pteridaceae (5 spp.). Os gêneros com maior número de espécies foram Nephrolepis, Polypodium e Thelypteris. Das 32 espécies registradas, 23 são ervas terrestres, três são ervas epífitas, três ervas aquáticas, duas arborescentes e uma erva hemiepífita. A maior riqueza de espécies e famílias foi registrada para a formação de mata periodicamente inundada e a menor, para a formação arbustiva fechada de pós-praia. Há baixa similaridade entre a flora pteridofítica de diferentes áreas de restingas analisadas e entre as restingas e a mata atlântica. Blechnum serrulatum e Polypodium triseriale são espécies de ocorrência comum em áreas de restingas. Das 32 espécies inventariadas, 17 possuem usos atribuídos (medicinais, ornamentais e comestíveis).
\end{abstract}

Palavras-chave: Pteridófitas, restinga, floresta atlântica, biodiversidade, etnobotânica

\begin{abstract}
Floristic analysis of the pteridophytes of the Restinga de Jurubatiba National Park, Rio de Janeiro, Brazil). The Restinga de Jurubatiba National Park is located on the Northern coast of Rio de Janeiro State (municipalities of Macaé, Carapebus and Quissamã). There are 12 families, 21 genera and 32 species, with the following families present: Blechnaceae, Cyatheaceae, Davalliaceae, Dennstaedtiaceae, Dryopteridaceae, Gleicheniaceae, Lycopodiaceae, Polypodiaceae, Pteridaceae, Salviniaceae, Schizaeaceae and Thelypteridaceae. The most species-rich families are Pteridaceae (5 spp.) and Polypodiaceae (5 spp.). Nephrolepis, Polypodium and Thelypteris were the richest genera. Of the 32 species recorded for the area, 23 are terrestrial herbs, three are epiphytes, three are aquatics, two are arborescent and one is a hemiepiphyte. The periodically flooded forest was the richest in species and families; the beach thicket presented the lowest values. The fern flora of several areas of restinga on the Brazilian coast showed low similarity values; this is also true for restingas and Atlantic forest. Blechnum serrulatum and Polypodium triseriale are commonly found in areas of restinga. Of the 32 species studied, 17 have economic potential (medicinal, ornamental and food).
\end{abstract}

Key words: Pteridophytes, sandy coastal plains, Atlantic forest, biodiversity, ethnobotany

\section{Introdução}

Denomina-se restinga ao conjunto formado pela deposição de sedimentos arenosos marinhos quaternários ao longo do litoral brasileiro e a biota que neles se instalou (Araujo \& Lacerda 1987; Araujo \& Maciel 1998). Sua vegetação, por estar localizada em áreas privilegiadas do litoral, está constantemente ameaçada pela especulação imobiliária e industrial (Araujo \& Lacerda 1987). Tornam-se, portanto, urgentes os trabalhos de reconhecimento de sua diversidade.

Segundo Tryon \& Tryon (1982), existem cerca de 9.000 espécies de pteridófitas no mundo, das quais cerca de 2.250 ocorrem nas Américas. Windisch (1992) estima que para as Américas ocorram cerca de 3.250 espécies, sendo que $30 \%$ deste montante pode ser encontrado no território brasileiro. As regióes Sul e Sudeste do Brasil contém cerca de 600 espécies e abriga um dos centros de endemismo e especiação de

Parte da Dissertação de Mestrado do primeiro Autor

2 Departamento de Ciências, Faculdade de Formação de Professores, Universidade do Estado do Rio de Janeiro, Rua Dr. Francisco Portela, 794, CEP 24435-000, São Gonçalo, RJ, Brasil

3 Departamento de Botânica, Instituto de Biologia, Universidade Federal Rural do Rio de Janeiro, BR 465 Km 7, CEP 23890-000, Seropédica, RJ, Brasil (lana@ufrrj.br)

4 Departamento de Ecologia, Instituto de Biologia, Universidade Federal do Rio de Janeiro, CCS/Bloco A, CEP 21941-590, Ilha do Fundão, Rio de Janeiro, RJ, Brasil

4 Autor para correspondência: mguerras@bol.com.br 
pteridófitas no Continente Sul-Americano (Tryon 1986). Para o Estado do Rio de Janeiro, apesar dos trabalhos florísticos realizados (Vellozo 1827; Brade 1942; Rizzini 1954; Mynssen 2000; Mynssen \& Sylvestre 2001; Mynssen et al. 2002; Santos \& Sylvestre 2001; Sylvestre 1997a; 1997b; Reis 1998; Marquete et al. 2001), ainda não existe uma estimativa do número de espécies. De acordo com Jermy (1990), as pteridófitas ocupam quase todos os hábitats, mas a maior riqueza de espécies é encontrada na floresta tropical úmida. Este autor alertou para o rápido desaparecimento destas florestas no mundo, com muitas de suas espécies ainda não descobertas.

Segundo Behar \& Viégas (1992) as restingas foram pouco estudadas no que se refere a levantamentos florísticos em pteridófitas. Trabalhos mais específicos para o grupo são raros, como o de Pontual (1972), que registrou 12 espécies para o Nordeste brasileiro; Barros et al. (1988) que registraram apenas duas espécies para as restingas do Estado de Pernambuco; para o Estado do Espírito Santo, Behar \& Viégas (1992) encontraram 31 espécies (Restinga de Setiba) e Behar \& Viégas (1993) 15 espécies (Restinga de Comboios). No Estado do Rio de Janeiro, Santos \& Sylvestre (2001) registraram 31 espécies para a Restinga de Jurubatiba.

O presente trabalho tem como objetivo contribuir para o conhecimento da riqueza de pteridófitas no Parque Nacional da Restinga de Jurubatiba. Visa também analisar o potencial econômico das espécies e a similaridade florística entre diversas áreas de restinga e mata atlântica ocorrentes no Sudeste/Sul do Brasil, indicando o ambiente preferencial ocupado por estas plantas.

\section{Material e métodos}

Área de estudo - O Parque Nacional da Restinga de Jurubatiba, criado em 29/abril/1998, está situado entre as coordenadas $22^{\circ}$ e $22^{\circ} 23^{\prime} \mathrm{S}$ e $41^{\circ} 15^{\prime}$ e $41^{\circ} 45^{\prime} \mathrm{W}$ (Fig. 1). A Restinga de Jurubatiba abrange três municípios, Macaé, Carapebus e Quissamã (Araujo et al. 1998).

A planície quaternária possui superfície relativamente plana, com altitude máxima de aproximadamente $12 \mathrm{~m}$ e inclina-se suavemente rumo ao oceano Atlântico. Entre as antigas cristas praiais encontram-se zonas inundáveis. A distribuição das chuvas é fortemente sazonal, com mínima mensal no inverno $(41 \mathrm{~mm})$ e máxima no verão $(189 \mathrm{~mm})$; a precipitação anual é de $1.100 \mathrm{~mm}$. A temperatura média anual é de $22,6^{\circ} \mathrm{C}$, com máxima em janeiro $\left(29,7^{\circ} \mathrm{C}\right)$ e mínima em julho $\left(20,0^{\circ} \mathrm{C}\right)$ (Araujo et al. 1998).

O solo arenoso, pobre em nutrientes, sempre foi um dos fatores limitantes ao estabelecimento de atividades agrícolas em áreas de restingas, e esse fato contribuiu para manter relativamente preservadas diversas comunidades vegetais na região de Macaé, séculos após o início da colonização. Entretanto, a exploração petrolífera da Bacia de Campos, iniciada em 1977, e a recente emancipação política dos distritos de Carapebus e Quissamã, estão dando início a grande e preocupante transformação desses ambientes (Araujo et al. 1998).

A classificação adotada para as formações vegetais da Restinga de Jurubatiba foi a proposta por Araujo et al. (1998): halófila-psamófila reptante, arbustiva fechada de pós-praia, arbustiva aberta de Clusia, arbustiva aberta de Ericaceae, mata periodicamente inundada, mata permanentemente inundada, mata de cordão arenoso, arbustiva aberta de Palmae, herbácea brejosa e aquática. A mata de cordão arenoso e a arbustiva aberta de Palmae não foram analisadas. Como áreas alteradas foram incluídas as margens do canal Macaé-Campos, as margens das estradas, áreas desmatadas e/ou queimadas e áreas inundadas dominadas por Typha domingensis Pers. (Typhaceae).

Coleta e tratamento do material botânico - Foram realizadas excursões à Restinga de Jurubatiba, no período

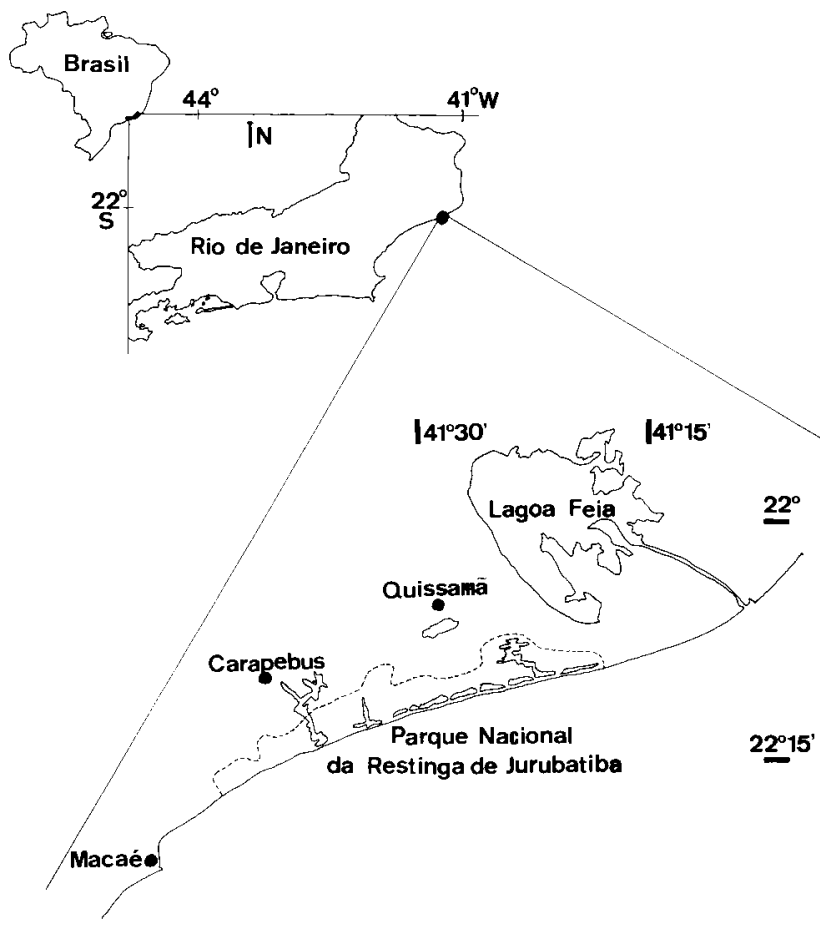

Figura 1. Mapa de localização do Parque Nacional da Restinga de Jurubatiba. 
de abril/1995 a maio/1999, com o total de 62 espécimes de pteridófitas coletados. As coletas foram concentradas nos arredores da estrada que liga a cidade de Carapebus à Praia da Capivara, na Fazenda São Lázaro e nos arredores da Lagoa de Cabiúnas. Todo o material botânico foi depositado no Herbário do Museu NacionalUFRJ (R). O estudo foi complementado com a análise de 73 exsicatas oriundas de coleções dos seguintes herbários: Museu Nacional-UFRJ (R); Herbário Alberto Castellanos-DECAM/FEEMA (GUA); Herbário do Instituto de Pesquisas Jardim Botânico do Rio de Janeiro (RB); Herbarium Bradeanum (HB) e Herbário da Universidade Santa Úrsula (RUSU).

Para a identificação do material utilizou-se bibliografia especializada. O sistema de classificação adotado foi o proposto por Tryon \& Tryon (1982), com modificações para o tratamento da família Lycopodiaceae (Øllgaard \& Windisch 1987) e Thelypteridaceae, com o reconhecimento do gênero Macrothelypteris. Para a família Cyatheaceae seguiu-se Lellinger (1987).

Os dados de distribuição geográfica foram baseados em referências bibliográficas e de levantamento das coleções oriundas dos Herbários $\mathrm{R}$ e RB, e os padrões adotados foram baseados em Lima et al. (1997). As informações referentes à utilidade das espécies foram obtidas por intermédio de bibliografia (Braga 1976; Sehnem 1979a; Sehnem 1979b; Sehnem 1979c; Corrêa 1984; Zurlo \& Brandão 1989; Roriz 1992; Lorenzi \& Souza 1995; Barros \& Andrade 1997; Santos \& Sylvestre 2000).

Uma planilha com as espécies de pteridófitas registradas para diferentes restingas brasileiras foi confeccionada para auxiliar as análises de distribuição apresentadas (Stellfeld 1949; Reitz 1961; Pontual 1972; Pfadenhauer 1978; Araujo \& Henriques 1984; Silva \& Somner 1984; Araujo \& Oliveira 1988; Barros et al. 1988; Silva \& Oliveira 1989; Behar \& Viégas 1992; 1993; Menezes-Silva 1998 e Santos \& Sylvestre 2001).

A caracterização do tipo de hábito e substrato adotada foi como se segue: arborescentes e ervas terrestres, epífitas, aquáticas e hemiepífitas.

$\mathrm{Na}$ análise comparativa da flora pteridofítica da Restinga de Jurubatiba com outras áreas de restinga (Behar \& Viégas 1992; 1993; Menezes-Silva 1998) e de mata atlântica (Mynssen \& Sylvestre 2001; Mynssen et al. 2002; Reis 1998; Sylvestre 1997a), utilizou-se o coeficiente de similaridade de Sørensen (Margurran 1988), por meio do programa FITOPAC. Este índice foi escolhido porque foram analisados dados qualitativos binários (presença-ausência). Esse índice valoriza a ocorrência simultânea de duas espécies e não é considerada a dupla-ausência (Valentin 1995). Para representar as relações de similaridade entre as diversas áreas analisadas foram utilizados dendrogramas.

\section{Resultados}

No levantamento das pteridófitas do Parque Nacional da Restinga de Jurubatiba foram registradas 12 famílias, 21 gêneros e 32 espécies (Tab. 1). Este resultado corresponde a $5,2 \%$ da flora vascular do Parque. As famílias com maior riqueza específica foram Polypodiaceae e Pteridaceae, com cinco espécies cada e Thelypteridaceae, com quatro espécies. As famílias com menor riqueza específica foram Lycopodiaceae, Gleicheniaceae e Blechnaceae, com uma espécie cada. A família que apresentou o maior número de gêneros foi Pteridaceae (Acrostichum, Ceratopteris, Doryopteris e Pityrogramma). As famílias com apenas um gênero foram Blechnaceae (Blechnum), Gleicheniaceae (Dicranopteris), Lycopodiaceae (Lycopodiella), Cyatheaceae (Cyathea) e Davalliaceae (Nephrolepis). Os gêneros com maior número de espécies foram Nephrolepis (N. biserrata, $N$. pectinata e $N$. rivularis), Polypodium (P. latipes, $P$. polypodioides e P. triseriale) e Thelypteris (T. interrupta, T. longifolia e T. serrata).

As ervas terrestres foram as mais representativas, com 23 espécies e as ervas hemiepífitas apresentaram apenas uma espécie (Tab. 1).

A mata periodicamente inundada foi a formação que apresentou o maior número de espécies de pteridófitas (18 espécies) e variações de tipos de hábito e substrato. Nesta formação ocorrem ervas terrestres (14 espécies), erva epífita (uma espécie), erva hemiepífita (uma espécie) e arborescentes (duas espécies) (Tab. 2). A formação com menor número de espécies foi a arbustiva fechada de pós-praia, com duas espécies (Tab. 2). Na formação halófita e psamófila reptante não foi registrada a presença de pteridófitas.

As espécies mais amplamente distribuídas entre as formações vegetais (incluindo as áreas alteradas) foram Blechnum serrulatum (ocorrendo em seis formações), Pteridium aquilinum (quatro), Microgramma vacciniifolia (quatro) e Polypodium triseriale (quatro). Das 32 espécies encontradas, 21 apresentaram ocorrência restrita a uma formação vegetal (Tab. 1).

De acordo com dados levantados de informações bibliográficas, Blechnum serrulatum e Polypodium 
Tabela 1. Padrões de distribuição geográfica e de formações vegetais das pteridófitas do Parque Nacional da Restinga de Jurubatiba, RJ. $\mathrm{MP}=$ mata periodicamente inundada; $\mathrm{MT}=$ mata permanentemente inundada; $\mathrm{AC}=$ arbustiva aberta de Clusia $; \mathrm{AE}=$ arbustiva aberta de Ericaceae; $\mathrm{PP}=$ arbustiva fechada de pós-praia; $\mathrm{HB}=$ herbácea brejosa; $\mathrm{VA}=$ vegetação aquática; $\mathrm{AA}=$ áreas alteradas. $\mathrm{PAN}=$ distribuição pantropical (regiões tropicais do velho e novo mundo); $\mathrm{NEO}=$ distribuição ampla na região neotropical (além dos limites da América do Sul); AS = distribuição limitada à América do Sul; BRA = distribuição limitada ao Brasil; RJ = distribuição limitada ao Rio de Janeiro, $\mathrm{X}=$ ocorrência do táxon; - = não há registro do táxon.

Famílias/Espécies

Formações vegetais

Distribuição

Hábito e substrato $\overline{M P}$ MT AC AE PP $\mathrm{HB}$ VA AA geográfica

\section{BLECHNACEAE}

Blechnum serrulatum Rich.

\section{CYATHEACEAE}

Cyathe a delgadii Sternb.

Cyathea microdonta (Desv.) Domin

DAVALLIACEAE

Nephrolepis biserrata (Sw.) Schott

Nephrolepis pectinata (Willd.) Schott

Nephrolepis rivularis (Vahl) Krug

DENNSTAEDTIACEAE

Lindsaea quadrangularis ssp. terminalis K.U. Kramer Lindsaea stricta var. stricta (Sw.) Dryand.

Pteridium aquilinum var. arachnoideum (Kaulf.) Brade DRYOPTERIDACEAE

Cyclodium meniscioides var. meniscioides (Willd.) C. Presl Erva terrestre

Polybotrya pilosa Brade

GLEICHENIACEAE

Dicranopteris flexuosa (Schrad.) Underw.

LYCOPODIACEAE

Lycopodiella alopecuroides var. integerrima (Spring)

B. Øllg. et P.G. Windisch

\section{POLYPODIACEAE}

Microgramma geminata (Schrad.) R. \& A. Tryon

Microgramma vacciniifolia (Langsd. et Fisch.) Copel.

Polypodium latipes Langsd. et Fisch.

Polypodium polypodioides var. minus (Fée) Weath.

Polypodium triseriale Sw.

PTERIDACEAE

Acrostichum danaeifolium Langsd. et Fisch.

Ceratopteris thalictroides (L.) Brongn.

Doryopteris collina (Raddi) J. Sm.

Pityrogramma calomelanos var. calomelanos (L.) Link

Pityrogramma trifoliata (L.) R.M. Tryon

SALVINIACEAE

Azolla caroliniana Willd.

Salvinia auriculata Aubl.

SCHIZAEACEAE

Lygodium volubile $\mathrm{Sw}$.

Schizaea elegans (Vahl) Sw.

Schizaea pennula Sw.

THELYPTERIDACEAE

Macrothelypteris torresiana (Gaud.) Ching

Thelypteris interrupta (Willd.) K. Iwats.

Thelypteris longifolia (Desv.) R.M. Tryon

Thelypteris serrata (Cav.) Alston

$\begin{array}{llllllllll}\text { Erva terrestre } & \mathrm{X} & \mathrm{X} & \mathrm{X} & \mathrm{X} & - & \mathrm{X} & - & \mathrm{X} & \mathrm{PAN} \\ \text { Arborescente } & \mathrm{X} & - & - & - & - & - & - & - & \text { NEO } \\ \text { Arborescente } & \mathrm{X} & - & - & - & - & - & - & - & \text { NEO } \\ & & & & & & & & & \\ \text { Erva terrestre } & \mathrm{X} & - & - & \mathrm{X} & - & - & - & - & \mathrm{PAN} \\ \text { Erva terrestre } & \mathrm{X} & - & - & \mathrm{X} & - & - & - & - & \text { NEO } \\ \text { Erva terrestre } & - & \mathrm{X} & - & - & - & - & - & - & \text { NEO } \\ & & & & & & & & & \\ \text { Erva terrestre } & \mathrm{X} & - & - & - & - & - & - & - & \text { AS } \\ \text { Erva terrestre } & - & - & - & - & - & X & - & - & \text { NEO } \\ \text { Erva terrestre } & \mathrm{X} & - & \mathrm{X} & \mathrm{X} & - & - & - & \mathrm{X} & \mathrm{NEO} \\ & & & & & & & & & \\ \text { Erva terrestre } & \mathrm{X} & - & - & - & - & - & - & - & \text { AS } \\ \text { Erva hemiepífita } & \mathrm{X} & - & - & - & - & - & - & - & \text { RJ } \\ \text { Erva terrestre } & - & - & - & - & - & - & - & X & \text { NEO } \\ \text { Erva terrestre } & - & - & - & - & - & X & - & - & \text { NEO }\end{array}$

Erva epífita

Erva epífita

Erva terrestre

Erva epífita

Erva terrestre

Erva terrestre

Erva aquática

Erva terrestre

Erva terrestre

Erva terrestre

Erva aquática

Erva aquática

Erva terrestre

Erva terrestre

Erva terrestre

$\begin{array}{ll}- & - \\ X & - \\ X & - \\ - & - \\ X & X\end{array}$

$\begin{array}{ll}X & X \\ X & X \\ - & - \\ - & - \\ X & X\end{array}$

BRA

NEO

NEO

AS

NEO

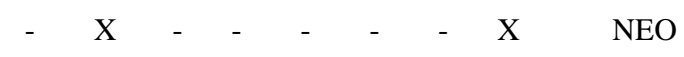

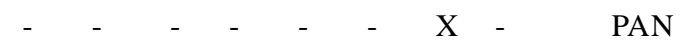

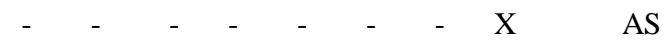

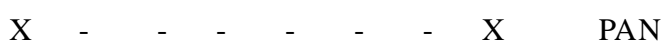

NEO

NEO

NEO

Erva terrestre

Erva terrestre

Erva terrestre

Erva terrestre
NEO

NEO

NEO

PAN

PAN

AS

NEO 
Tabela 2. Riqueza de espécies, de hábito e substrato por formação vegetal, no Parque Nacional da Restinga de Jurubatiba, RJ.

\begin{tabular}{|c|c|c|c|c|c|}
\hline \multirow[t]{2}{*}{ Formação vegetal } & \multicolumn{4}{|c|}{ Substrato } & \multirow[t]{2}{*}{ Tota } \\
\hline & Terrestre & Epífita & Aquática & Hemiepífita & \\
\hline Mata periodicamente inundada & $16^{*}$ & 1 & - & 1 & 18 \\
\hline Arbustiva aberta de Ericaceae & 7 & 2 & - & - & 9 \\
\hline Áreas alteradas & 8 & - & - & - & 8 \\
\hline Mata permanentemente inundada & 7 & - & - & - & 7 \\
\hline Arbustiva aberta de Clusia & 3 & 2 & - & - & 5 \\
\hline Herbácea brejosa & 3 & - & - & - & 3 \\
\hline Vegetação aquática & - & - & 3 & - & 3 \\
\hline Arbustiva fechada de pós-praia & - & 2 & - & - & 2 \\
\hline
\end{tabular}

* inclui duas espécies arborescentes.

triseriale são as espécies mais amplamente distribuídas entre as restingas brasileiras.

Do número total de espécies inventariadas, 17 possuem usos atribuídos. Destes, 14 são consideradas medicinais, cinco ornamentais e duas são comestíveis (Tab. 3).

A análise da distribuição geográfica das espécies revelou cinco padrões. A maioria das espécies (59\%) apresenta uma ampla distribuição na região neotropical, o que representa 19 das 32 espécies inventariadas. Com distribuição pantropical foram registradas seis espécies (19\%) e cinco espécies (16\%) mostram-se

Tabela 3. Potencial econômico atribuído às espécies de pteridófitas do Parque Nacional da Restinga de Jurubatiba, RJ.

\begin{tabular}{llcl}
\hline \multirow{2}{*}{ Espécies } & \multicolumn{3}{c}{ Potencial econômico } \\
\cline { 2 - 4 } & Medicinal & Ornamental & Comestível \\
\hline Blechnum serrulatum & $\mathrm{X}$ & - & - \\
Cyathea microdonta & $\mathrm{X}$ & - & - \\
Nephrolepis biserrata & $\mathrm{X}$ & $\mathrm{X}$ & - \\
Nephrolepis pectinata & - & $\mathrm{X}$ & - \\
Pteridium aquilinum & $\mathrm{X}$ & - & $\mathrm{X}$ \\
$\quad$ var. arachnoideum & & & \\
Dicranopteris flexuosa & $\mathrm{X}$ & - & - \\
Lycopodiella alopecuroides & $\mathrm{X}$ & - & - \\
Microgramma vacciniifolia & $\mathrm{X}$ & - & - \\
Polypodium polypodioides & $\mathrm{X}$ & - & - \\
$\quad$ var. minus & & & \\
Polypodium triseriale & $\mathrm{X}$ & $\mathrm{X}$ & - \\
Acrostichum danaeifolium & $\mathrm{X}$ & $\mathrm{X}$ & - \\
Ceratopteris thalictroides & - & - & $\mathrm{X}$ \\
Pityrogramma calomelanos & $\mathrm{X}$ & - & - \\
$\quad$ var. calomelanos & & & \\
Azolla caroliniana & $\mathrm{X}$ & - & - \\
Salvinia auriculata & $\mathrm{X}$ & - & - \\
Lygodium volubile & - & $\mathrm{X}$ & - \\
Schizaea elegans & $\mathrm{X}$ & - & - \\
Total & 14 & 5 & 2 \\
\hline & & & \\
\hline & & 5 & \\
\hline
\end{tabular}

limitadas à América do Sul. Apenas duas espécies (Microgramma geminata e Polybotrya pilosa) apresentam distribuição limitada ao Brasil, sendo a última restrita ao Estado do Rio de Janeiro (Tab. 1).

$\mathrm{Na}$ análise da similaridade da flora pteridofítica entre as formações vegetais da Restinga de Jurubatiba (Fig. 2), existe um grupo constituído pelas formações arbustiva aberta de Ericaceae, arbustiva aberta de Clusia, mata periodicamente inundada, mata permanentemente inundada e áreas alteradas. As formações herbácea brejosa, arbustiva fechada de pós-praia e vegetação aquática formaram grupos isolados.

As formações que apresentaram similaridade superior a $50 \%$ foram arbustiva aberta de Clusia e

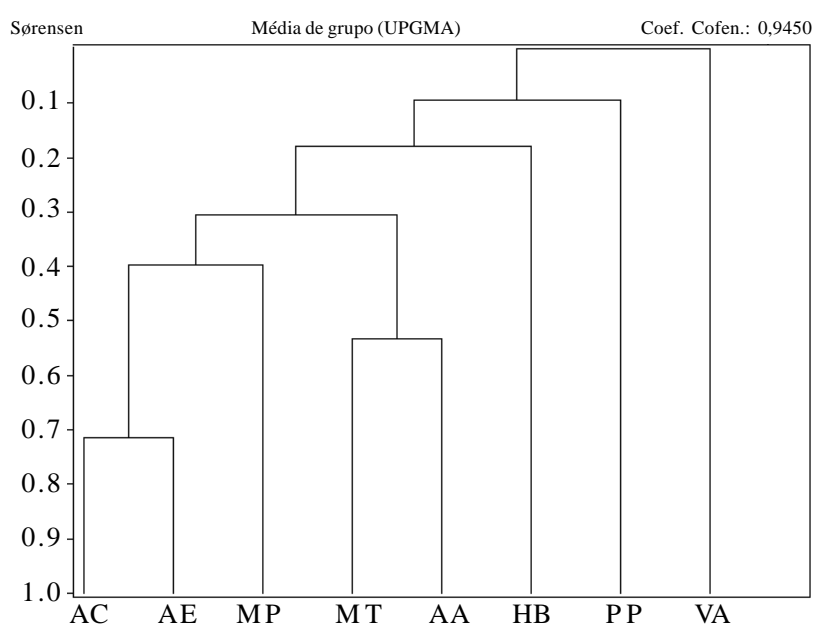

Figura 2. Dendrograma mostrando a similaridade da flora pteridofítica entre as formações vegetais do Parque Nacional da Restinga de Jurubatiba. MP = mata periodicamente inundada; $\mathrm{MT}=$ mata permanentemente inundada $\mathrm{AC}=$ arbustiva aberta de Clusia; $\mathrm{AE}=$ arbustiva aberta de Ericaceae; $\mathrm{PP}=$ arbustiva fechada de pós-praia; $\mathrm{HB}$ = herbácea brejosa; $\mathrm{VA}$ = vegetação aquática; $\mathrm{AA}=$ áreas alteradas. 
arbustiva aberta de Ericaceae (> 70\%) e mata permanentemente inundada e áreas alteradas (> 50\%) (Fig. 2).

$\mathrm{Na}$ análise da similaridade entre a flora pteridofítica de algumas áreas de restinga e mata atlântica, foram detectados três grupos (Fig. 3). O primeiro formado pelas áreas de restinga (Jurubatiba, Setiba, Comboios e Ilha do Mel), o segundo pelas áreas de mata atlântica (ou florestas de baixada e baixo montanas - Poço das Antas, Arboreto do Jardim Botânico, Horto Florestal e Mundo Novo) e um terceiro grupo formado pela região de Macaé de Cima (mata atlântica de altitude ou florestas alto montanas). Há baixa similaridade $(<20 \%)$ entre a flora pteridofítica das restingas e da mata atlântica.

Na Tabela 4, comparou-se o número de espécies por tipo de hábito e substrato da Restinga de Jurubatiba com uma área de restinga no Estado do Espírito Santo (Setiba), uma de mata atlântica alto montana (Serra de Macaé de Cima) e uma de mata atlântica montana (Rio das Pedras). As restingas de Jurubatiba e de Setiba, assim como Rio das Pedras, apresentam elevado número de ervas terrestres e baixo número de epífitas. Contrariamente, a Serra de Macaé de Cima possui percentagem maior de epífitas em relação às ervas terrestres (Tab. 4).

Comparando a riqueza de espécies por formação vegetal do Parque Nacional da Restinga de Jurubatiba com outras áreas de restinga (Setiba e Comboios), observou-se que a formação de mata periodicamente inundada possui a maior riqueza de espécies em todas as áreas, e a arbustiva fechada de pós-praia, a menor riqueza (Tab. 5).

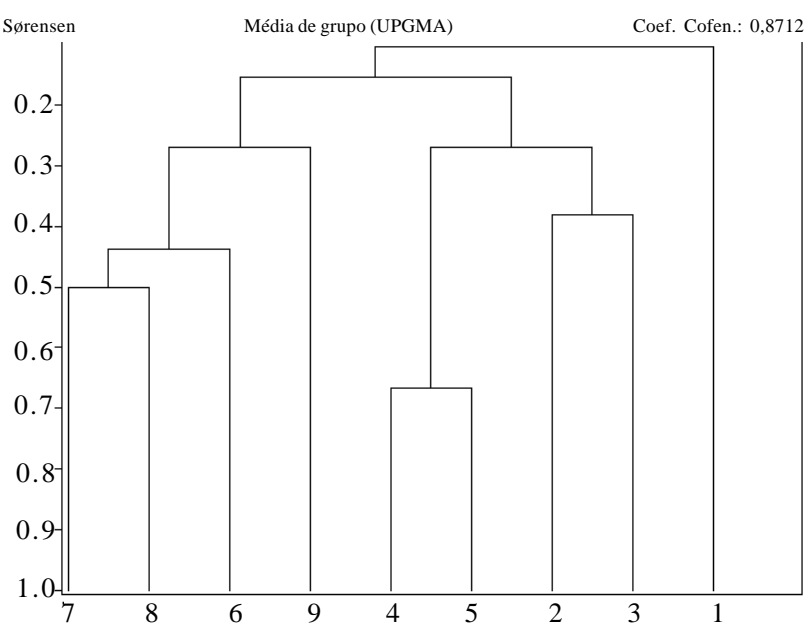

Figura 3. Dendrograma mostrando a similaridade da flora pteridofítica entre diferentes áreas de restinga e mata atlântica. 1 - Reserva Ecológica de Macaé de Cima-RJ; 2 - Reserva Biológica de Poço das Antas-RJ; 3 - Mata de encosta do arboreto do Jardim Botânico do Rio de Janeiro-RJ; 4 - Mata de encosta do Horto Florestal-RJ; 5 - Mata de encosta da vertente leste do Morro Mundo Novo-RJ; 6 - Parque Nacional da Restinga de Jurubatiba-RJ; 7 - Parque Estadual de Setiba-ES; 8 - Restinga de Comboios-ES; 9 - Ilha do Mel-PR

Tabela 4. Comparação entre o número de espécies por hábito e substrato do Parque Nacional da Restinga de Jurubatiba (RJ), Restinga de Setiba (ES), Macaé de Cima (mata atlântica, RJ) e Reserva Rio das Pedras (mata atlântica, RJ).

\begin{tabular}{|c|c|c|c|c|c|c|c|c|c|}
\hline \multirow[t]{2}{*}{ Hábito } & \multirow[t]{2}{*}{ Substrato } & \multicolumn{2}{|c|}{ Jurubatiba } & \multicolumn{2}{|c|}{ Setiba $^{1}$} & \multicolumn{2}{|c|}{ Macaé de Cima² } & \multicolumn{2}{|c|}{ Rio das Pedras ${ }^{3}$} \\
\hline & & N. espécies & $\%$ & N. espécies & $\%$ & N. espécies & $\%$ & N. espécies & $\%$ \\
\hline Arborescente & Terrestre & 2 & 6,3 & 1 & 3,3 & 8 & 10,0 & 3 & 2,6 \\
\hline \multirow[t]{5}{*}{ Erva } & Terrestre & 23 & 71,9 & 20 & 66,7 & 29 & 36,3 & 59 & 51,8 \\
\hline & Epífita & 3 & 9,4 & 4 & 13,4 & 39 & 48,7 & 21 & 18,4 \\
\hline & Aquática & 3 & 9,4 & 2 & 6,7 & - & - & - & - \\
\hline & Hemiepífita & 1 & 3,1 & 2 & 6,7 & 1 & 1,3 & 5 & 4,4 \\
\hline & Rupícola & - & - & 1 & 3,3 & 3 & 3,7 & 26 & 22,8 \\
\hline Total & & 32 & 100 & 30 & 100 & 80 & 100 & 114 & 100 \\
\hline
\end{tabular}

${ }^{1}$ Behar \& Viégas (1992); ${ }^{2}$ Sylvestre (1997a); ${ }^{3}$ Mynssen (2000).

\section{Discussão}

Segundo Araujo et al. (2001), foram registradas 588 espécies de angiospermas para o Parque Nacional da Restinga de Jurubatiba que, juntamente com as pteridófitas, totalizam 620 espécies vasculares. As pteridófitas correspondem, portanto, a 5,2\% das espécies da flora vascular da Restinga de Jurubatiba. Na Ilha do Mel, PR (restinga), essa representatividade foi de 10,9\% (Menezes-Silva 1998).

A maioria das espécies registradas para a Restinga de Jurubatiba possui distribuição ampla na região neotropical (59\%). Resultados similares foram encontrados para outras restingas e regiões de mata 
Tabela 5. Comparação da riqueza de espécies por formação vegetal do Parque Nacional da Restinga de Jurubatiba, Restinga de Setiba (ES) e Restinga de Comboios (Aracruz/Linhares, ES). Dados percentuais.

\begin{tabular}{lccc}
\hline \multirow{2}{*}{ Formação Vegetal } & \multicolumn{2}{c}{ RJ } & \multicolumn{2}{c}{ ES } \\
\cline { 2 - 4 } & Jurubatiba Setiba ${ }^{1}$ Comboios $^{2}$ \\
\hline Mata periodicamente inundada & 32,7 & 28,1 & 42,9 \\
Mata do cordão arenoso & - & 22,8 & 19,0 \\
Arbustiva aberta de Ericaceae & 16,4 & 17,5 & - \\
Áreas alteradas & 14,5 & - & - \\
Mata permanentemente inundada & 12,7 & 5,3 & - \\
Arbustiva aberta de Clusia & 9,1 & 8,8 & 9,5 \\
Herbácea brejosa & 5,5 & 8,8 & 23,8 \\
Vegetação aquática & 5,5 & 3,5 & 4,8 \\
Arbustiva fechada de pós-praia & 3,6 & 1,8 & - \\
Afloramento rochoso & - & 3,5 & - \\
Total & 100 & 100 & 100 \\
\hline
\end{tabular}

${ }^{1}$ Behar \& Viégas (1992); ${ }^{2}$ Behar \& Viégas (1993).

atlântica. Em Macaé de Cima, este padrão corresponde a 46,2\% das espécies inventariadas (Sylvestre 1997a); na REBIO Poço das Antas, a 59\% (Reis 1998); nos morros litorâneos do município do Rio de Janeiro, Morro Mundo Novo e encosta do Jardim Botânico do Rio de Janeiro, este padrão corresponde, respectivamente, a 40\% e 51,9\% das espécies (Mynssen \& Sylvestre 2001; Mynssen et al. 2002); na Restinga de Setiba, 44,8\% (Behar \& Viégas 1992) e para a Restinga de Comboios, 53,3\% (Behar \& Viégas 1993). A ampla distribuição de algumas espécies de pteridófitas corresponderia à maior facilidade de seus esporos serem dispersados no ar e permanecerem viáveis por período considerável, podendo ser dispersos por longas distâncias, aliado a fenótipo com grande amplitude ecológica (Tryon 1970).

As formações que apresentaram maior riqueza de espécies foram a mata periodicamente inundada, que também apresentou maior número de famílias, hábito e substrato, e a arbustiva aberta de Ericaceae. $\mathrm{Na}$ primeira, o lençol freático aflora na estação chuvosa, o solo apresenta grande quantidade de serrapilheira e há menor intensidade luminosa no bosque e subosque da mata. Na segunda, o lençol freático é mais superficial, onde há o domínio de Bonnetia stricta - Theaceae (Araújo et al. 1998). As pteridófitas necessitam da água para a reprodução no seu ciclo vital. Deste modo, são preferencialmente encontradas em ambientes ou substratos que possam reter água ao menos parte do tempo, apesar de existirem espécies adaptadas aos mais diversos hábitats, inclusive em lugares secos como as caatingas nordestinas (Windisch 1992).

As formações que apresentaram menor riqueza de espécies foram a arbustiva fechada de pós-praia (2), a herbácea brejosa (3) e a vegetação aquática (3). A disponibilidade hídrica na primeira formação é baixa e na segunda, há variações extremas, podendo estar seca ou totalmente encharcada (Araujo et al. 1998). Portanto, as espécies que ocorrem nestas formações precisam ter estratégias para suportar as variações hídricas. Polypodium polypodioides, epífita poiquiloídrica (Benzing 1990) ocorre na formação arbustiva fechada de pós-praia. Esta espécie apresenta enrolamento das lâminas, que é um processo utilizado para regulação hídrica (Larcher 2000; Waechter 1992), e também possui densa camada de escamas sobre a folha, outra estratégia importante para esta função (Fontoura et al. 1997). A estratégia da Microgramma vacciniifolia, que também ocorre na formação pós-praia, é apresentar rizoma suculento (Fontoura et al. 1997; Larcher 2000). Das espécies que ocorrem na formação herbácea brejosa, Lycopodiella alopecuroides e Linsaea stricta, possuem como adaptação às variações hídricas, as folhas reduzidas (Larcher 2000; Waechter 1992) e Blechnum serrulatum possui estrutura escleromorfa (folhas espessas e rígidas).

Observou-se que as espécies mais amplamente distribuídas, entre as formações vegetais na Restinga de Jurubatiba e as diferentes restingas analisadas, foram Blechnum serrulatum e Polypodium triseriale. Ambas possuem estratégias de regulação hídrica. Blechnum serrulatum tem estrutura escleromorfa, já mencionada, enquanto Polypodium triseriale possui, além do rizoma suculento, a possibilidade da abscisão das folhas em períodos desfavoráveis, o que pode ser constatado pelos pecíolos articulados ao caule (Larcher 2000).

A mata periodicamente inundada apresenta apenas uma epífita (Microgramma vacciniifolia) que é encontrada às margens desta formação. $\mathrm{O}$ reduzido número de epífitas pode estar sendo determinado pelo tipo de substrato, de hospedeiro ou condições de umidade (Fontoura et al. 1997).

As fomações arbustiva aberta de Ericaceae e de Clusia possuem a mesma estrutura de vegetação, ou seja em moitas. Portanto, devem ter condições análogas de irradiância, o que pode explicar a similaridade da flora pteridofítica entre estas duas formações. Entretanto, as condições hídricas entre elas são diferentes, sendo o substrato da arbustiva aberta de Ericaceae mais úmido que o de Clusia. 
Foram incluídas dentro das áreas alteradas os alagados dominados por Typha domingensis, o que justifica a similaridade entre estas áreas e a mata permanentemente inundada.

A Restinga de Jurubatiba apresenta baixo número de pteridófitas epífitas, quando comparada com áreas de mata atlântica localizada no Estado do Rio de Janeiro (Macaé de Cima e Rio das Pedras) e outras regiões de vegetação litorânea do Brasil (Waechter 1992; Labiak \& Prado 1998). Verificou-se que o índice de chuvas para a Restinga de Jurubatiba é de 1.100 a $1.300 \mathrm{~mm}$ ao ano com um período seco entre os meses de julho e setembro e que a altitude máxima é de $12 \mathrm{~m}$ (Araujo et al. 1998). O volume de chuvas, o período seco, a altitude, assim como a latitude, são fatores que influenciam na abundância e diversidade de epífitas de uma região (Waechter 1992; Fontoura et al. 1997).

Apesar da baixa similaridade entre as restingas e as áreas de mata atlântica examinadas, observa-se que das espécies inventariadas na Restinga de Jurubatiba, apenas Acrostichum danaeifolium, Azolla carolliniana, Ceratopteris thalictroides e Salvinia auriculata, espécies aquáticas, não são comuns à mata atlântica. Provavelmente, estas espécies não encontram ambiente propício para se estabelecerem neste ambiente. Não foram registradas pteridófitas de ocorrência exclusiva para a Restinga de Jurubatiba. As restingas são consideradas formações associadas à mata atlântica (Pinto et al. 1996), sendo que muitas de suas espécies são originárias desta formação vegetal (Rizzini 1979; Araujo 2000).

Dentro do grupo formado pelas restingas, os resultados demonstram que não existe flora pteridofítica comum às restingas analisadas. As áreas que apresentaram maior similaridade foram as duas do Espírito Santo, Setiba e Comboios (50\%), provavelmente devido à proximidade geográfica. Além disso, as restingas de Comboios e Setiba (Espírito Santo) e Jurubatiba (Rio de Janeiro) formam uma unidade bem definida, com mais de $40 \%$ de similaridade, enquanto a restinga da Ilha do Mel (PR) tem ligação mais fraca com este grupo. Tryon (1986) afirma que as relações entre as floras pteridofíticas, de diferentes regiões, são geralmente dependentes das proximidades de áreas geográficas. Estudos focalizando as Angiospermas têm demonstrado que há heterogeneidade entre as floras das restingas brasileiras, e que fatores como pluviosidade, temperatura e geomorfologia podem contribuir para a diferenciação florística das mesmas (Araujo 2000).

\section{Agradecimentos}

À Coordenação de Aperfeiçoamento de Pessoal de Nível Superior (CAPES), pela bolsa concedida ao primeiro Autor; à coordenação do curso de Pós-graduação em Botânica do Museu Nacional do Rio de Janeiro; ao Dr. Paulo Günter Windisch, pelas críticas e sugestões; às pteridólogas Claudine Massi Mynssen e Fabiana Regina Nonato, pelo constante apoio.

\section{Referências bibliográficas}

Araujo, D.S.D. 2000. Análise florística e fitogeográfica das restingas do Estado do Rio de Janeiro. Tese de Doutorado. Universidade Federal do Rio de Janeiro, Rio de Janeiro.

Araujo, D.S.D. \& Henriques, R.P.B. 1984. Análise florística das Restingas do Estado do Rio de Janeiro. Pp. 159-193. In: L.D. Lacerda; D.S.D. Araujo; R. Cerqueira \& B. Turcq (orgs.). Restingas: origens, estruturas, processos. CEUFF, Niterói.

Araujo, D.S.D. \& Lacerda, L.D. 1987. A Natureza das Restingas. Ciência Hoje 6(33): 42-48.

Araujo, D.S.D. \& Oliveira, R.R. 1988. Reserva Biológica Estadual da Praia do Sul (Ilha Grande, Estado do Rio de Janeiro): Lista Preliminar da Flora. Acta Botanica Brasilica 1(2): 83-94, supl.

Araujo, D.S.D. \& Maciel, N.C. 1998. Restingas Fluminenses: Biodiversidade e Preservação. Boletim da Fundação Brasileira para a Conservação da Natureza 25: 27-51.

Araujo, D.S.D.; Scarano, F.R.; Sá, C.F.C.; Kurtz, B.C.; Zaluar, H.L.T.; Montezuma, R.C.M. \& Oliveira, R.C. 1998. Comunidades Vegetais do Parque Nacional da Restinga de Jurubatiba. Pp. 39-62. In: F.A. Esteves (ed.). Ecologia das lagoas costeiras do Parque Nacional da Restinga de Jurubatiba e do Município de Macaé. Rio de Janeiro. Universidade Federal do Rio de Janeiro, Rio de Janeiro.

Araujo, D.S.D.; Costa, A.F. da; Oliveira, A.S. \& Moura, R.L. 2001. Florística e padrões fitogeográficos. Pp. 155-165. In: A.F. Costa \& I.C.A. Dias. Flora do Parque Nacional da Restinga de Jurubatiba e arredores, Rio de Janeiro, Brasil: listagem, florística e fitogeografia: angiospermas, pteridófitas, algas continentais. Museu Nacional (Série Livros n. 8), Rio de Janeiro.

Barros, I.C.L. \& Andrade, L.H.C. 1997. Pteridófitas medicinais (samambaias, avencas e plantas afins). Ed. Universitária da Universidade Federal de Pernambuco, Recife.

Barros, I.C.L.; Lira, O.C. \& Silva, A.J.R. 1988. Distribuição geográfica das pteridófitas ocorrentes no Estado de Pernambuco, Brasil. Acta Botanica Brasilica 2(1-2): 47-84.

Behar, L. \& Viégas, G.M.F. 1992. Pteridophyta da Restinga do Parque Estadual de Setiba, Espírito Santo. Boletim do Museu de Biologia Mello Leitão 1: 39-59. 
Behar, L. \& Viégas, G.M.F. 1993. Pteridophyta da Restinga de Comboios, Aracruz/Linhares-ES. Pp. 134-143. In: Anais do III Simpósio de Ecossistemas da Costa Brasileira: subsídios a um gerenciamento ambiental, Serra Negra, SP. v. 3. ACIESP, São Paulo.

Benzing, D.H. 1990. Vascular epiphytes. Cambridge, University Press.

Brade, A.C. 1942. A composição da flora pteridófita do Itatiaia. Contribuição à fitogeografia dessa região. Rodriguésia 6(15): 29-43.

Braga, R. 1976. Plantas do Nordeste, especialmente do Ceará. $3^{\mathrm{a}}$ ed. Mossoró, Escola Superior de Agricultura.

Corrêa, M.P. 1984. Dicionário das plantas úteis do Brasil e das exóticas cultivadas. $2^{\mathrm{a}} \mathrm{ed}$. Instituto Brasileiro de Desenvolvimento Florestal, Rio de Janeiro. v. 1-6.

Fontoura, T.; Sylvestre, L.S.; Vaz, A.M.S.F. \& Vieira, C.M. 1997. Epífitas vasculares, hemiepífitas e hemiparasitas da Reserva Ecológica de Macaé de Cima. Pp. 89-101. In: H.C. Lima \& R.R. Guedes-Bruni (eds.). Serra de Macaé de Cima: Diversidade Florística e Conservação em Mata Atlântica. Jardim Botânico do Rio de Janeiro, Rio de Janeiro.

Jermy, A.C. 1990. Conservation of Pteridophytes Pp. 145-152. In: K. Kubitzki (ed.). The families and genera of vascular plants. K.U. Kramer \& P.S. Green (eds.). Pteridophytes and Gymnosperms. Springer-Verlag, Berlin, v. 1.

Labiak, P. H. \& Prado, J. 1998. Pteridófitas epífitas da Reserva Volta Velha, Itapoá - Santa Catarina, Brasil. Boletim do Instituto de Botânica 11: 1-79.

Larcher, W. 2000. Ecofisiologia vegetal. RiMa, São Carlos.

Lellinger, D.B. 1987. The disposition of Trichopteris (Cyatheaceae). American Fern Journal 77(3): 90-94.

Lima, M.P.M.; Guedes-Bruni, R.R.; Sylvestre, L.S.; Pessoa, S.A.V.; Andreata, R.H.P. 1997. Padrões de distribuição geográfica das espécies vasculares da Reserva Ecológica de Macaé de Cima. Pp. 103-122. In: H.C. Lima \& R.R. Guedes-Bruni (eds.). Serra de Macaé de Cima: Diversidade Florística e Consevação em Mata Atlântica. Jardim Botânico do Rio de Janeiro, Rio de Janeiro.

Lorenzi, H. \& Souza, H.M. 1995. Plantas ornamentais no Brasil: arbustivas, herbáceas e trepadeiras. Editora Plantarum, Nova Odessa.

Margurran, A.E. 1988. Ecological diversity and its measurement. Princeten University Press, Princeton.

Marquete, R.; Valente, M.C.; Silva, N.M.F.; Guimarães, E.F.; Marquete, O.; Giordano, L.C.S.; Vaz, A.M.S.F.; Quinet, A.; Silva, C.N.A.; Macedo, P.B.; Munssen, C.; Pinto, C.G.; Pinheiro, F.C.; Garcia, R.O. \& Medeiros, E. von S. 2001. Checklist das espécies ocorrentes nas áreas do entorno do Instituto de Pesquisas Jardim Botânico do Rio de Janeiro - Pteridófitas e Angiospermas. Bradea 37(7): 227-258.

Menezes-Silva, S. 1998. As formações vegetais da planície litorânea da Ilha do Mel, Paraná: composição florística e principais características estruturais. Tese de Doutorado. Universidade Estadual de Campinas, Campinas.
Mynssen, C.M. 2000. Pteridófitas da Reserva Rio das Pedras, Mangaratiba, RJ. Dissertação de Mestrado. Museu Nacional da Universidade Federal do Rio de Janeiro.

Mynssen, C.M. \& Sylvestre, L.S. 2001. Pteridófitas do Morro Mundo Novo, Rio de Janeiro, RJ. Eugeniana 25: 26-31.

Mynssen, C.M.; Sylvestre, L.S. \& Andreata, R.H.P. 2002. Pteridófitas das matas de encosta do Jardim Botânico do Rio de Janeiro. Pesquisas, botânica 52: 47-87.

Øllgaard, B. \& Windisch, P.G. 1987. Sinopse das Licopodiáceas do Brasil. Bradea 5(1): 1-43.

Pfadenhauer, J. 1978. Contribuição ao conhecimento da vegetação e de suas condições de crescimento nas dunas costeiras do Rio Grande do Sul, Brasil. Revista Brasileira de Biologia 38(4): 827-836.

Pinto, L.P.S.; Costa, J.P.O.; Fonseca, G.A.B. \& Costa, C.M.R. 1996. Mata Atlântica: ciência, conservação e políticas. In: Workshop Científico sobre a Mata Atlântica, Belo Horizonte, MG. Documentos Ambientais. 23 p.

Pontual, I.B. 1972. Pteridófitas do Nordeste. Pp. 41-43. In: Anais do XXIII Congresso Nacional de Botânica. Garanhuns 1972. Sociedade Botânica do Brasil, Garanhuns.

Reis, C.A. 1998. Pteridófitas da Reserva Biológica de Poço das Antas, Município de Silva Jardim - Rio de Janeiro: Lista das espécies e Análise florística. Monografia de Graduação. Universidade Federal Rural do Rio de Janeiro, Rio de Janeiro.

Reitz, R. 1961. Vegetação da zona marítima de Santa Catarina. Sellowia 13: 17-115.

Rizzini, C.T. 1954. Flora Organensis. Arquivos do Jardim Botânico do Rio de Janeiro 13: 118-243.

Rizzini, C.T. 1979. Tratado de fitogeografia do Brasil. Aspectos sociológicos e florísticos. v. 2. Hucitec, São Paulo.

Roriz, A. 1992. Avencas e samambaias. Sítios \& Jardins 8: 1-71.

Santos, M.G. \& Sylvestre, L.S. 2000. Pteridófitas comercializadas por erveiros de Niterói e do Rio de Janeiro, RJ, Brasil: uma abordagem etnobotânica. Leandra 15: 79-90.

Santos, M.G. \& Sylvestre, L.S. 2001. Pteridófitas. Pp. 143-152. In: A.F. Costa \& I.C.A. Dias. Flora do Parque Nacional da Restinga de Jurubatiba e arredores, Rio de Janeiro, Brasil: listagem, florística e fitogeografia: angiospermas, pteridófitas, algas continentais. Museu Nacional (Série Livros n. 8), Rio de Janeiro.

Sehnem, A. 1979a. Davaliáceas. Pp. 1-18. In: R. Reitz (ed.). Flora Ilustrada Catarinense. Herbário Barbosa Rodrigues, Itajaí.

Sehnem, A. 1979b. Parkeriáceas. Pp. 1-5. In: R. Reitz (ed.). Flora Ilustrada Catarinense. Herbário Barbosa Rodrigues, Itajaí.

Sehnem, A. 1979c. Salviniáceas. Pp. 1-11. In: R. Reitz (ed.). Flora Ilustrada Catarinense. Herbário Barbosa Rodrigues, Itajaí.

Silva, J.G. \& Oliveira, A.S. 1989. A vegetação de Restinga no Município de Maricá, RJ (1). Acta Botanica Brasilica 3(2): 253-272, supl. 
Silva, J.G. \& Somner, G.V. 1984. A vegetação de Restinga na Barra de Maricá, RJ. Pp. 217-224. In: L.D. Lacerda; D.S.D. Araujo; R. Cerqueira \& B. Turcq (orgs.). Restingas: origens, estruturas, processos. CEUFF, Niterói.

Stellfeld, C. 1949. Aspectos da zona da praia do Estado do Paraná (Brasil). Lilloa 20: 203-214.

Sylvestre, L.S. 1997a. Pteridófitas da Reserva Ecológica de Macaé de Cima. Pp. 41-52. In: H.C. Lima \& R.R. GuedesBruni (eds.). Serra de Macaé de Cima: Diversidade Florística e Conservação em Mata Atlântica. Instituto de Pesquisas Jardim Botânico do Rio de Janeiro, Rio de Janeiro.

Sylvestre, L.S. 1997b. Pteridophyta. Pp. 44-49. In: M.C.M. Marques (coord.). Mapeamento da cobertura vegetal e listagem das espécies ocorrentes na Área de Proteção Ambiental de Cairuçu, município de Parati, RJ. Instituto de Pesquisas Jardim Botânico do Rio de Janeiro (Série Estudos e Contribuições número 13), Rio de Janeiro.

Tryon, R. 1970. Development and Evolution of Fern Floras of Oceanic Islands. Biotropica 2(2): 76-84.
Tryon, R.M. 1986. The Biogeography of Species, with Special Reference to Ferns. The Botanical Review 52(2): 117-155.

Tryon, R.M. \& Tryon, A.F. 1982. Ferns and allied plants with special reference to Tropical America. SpringerVerlag.

Valentin, J.L. 1995. Agrupamento e ordenação. Pp. 27-55. In: P.R. Peres-Neto; J.L. Valentin \& F.A.S. Fernandez (eds.). Oecologia brasiliensis volume II: Tópicos em tratamento de dados biológicos. Programa de Pósgraduação em Ecologia - Instituto de Biologia - UFRJ, Rio de Janeiro.

Vellozo, J.M. 1827. Florae Fluminensis Icones... Paris, v. 11.

Waechter, J.L. 1992. O epifitismo vascular na planície costeira do Rio Grande do Sul. Tese de Doutorado. Universidade Federal de São Carlos, São Carlos.

Windisch, P.G. 1992. Pteridófitas da região Norte-ocidental do Estado de São Paulo (Guia para estudo e excursões). $2^{\mathrm{a}}$ ed. UNESP, São José do Rio Preto.

Zurlo, C. \& Brandão, M. 1989. As ervas comestíveis: descrição, ilustração e receitas. Globo, Rio de Janeiro. 